\title{
Multi-Ingredient Pre-Workout Supplements and Energy Drinks
}

\author{
Tanya Bhasin
}

Student, Dept. Amity Institute of Food Technology, Amity University, Noida, India

*Corresponding Author: Tanya Bhasin

Email: bhasin.tanya99@gmail.com

\begin{abstract}
Human life is very precious therefore keeping in mind we should lead a happy and a healthy lifestyle. Life will only be happy if one is healthy therefore intake of Supplements and Energy drinks both are harmful for the body. Quantity should be kept in mind. Excess of everything has a bad affect on health.
\end{abstract}

Keywords: Multi ingredients pre workout supplements, Energy drinks.

\section{Introduction}

Various fixing pre-exercise supplements (MIPS) are indicated to prompt intense metabolic and physiological changes that may result in good body sythesis enhancements, for example, lower muscle to fat ratio and expanded slender weight, after some time. The utilization of dietary enhancements to improve execution is ending up progressively prevalent among competitors and wellness lovers. Shockingly, there is an enormous absence of research being finished in regards to female competitors and the utilization of game enhancements. As of late, another class of dietary enhancements called multi-fixing preexercise supplements (MIPS) has expanded in notoriety. These enhancements are expected to be taken preceding activity and regularly contain a mix of fixings, for example, caffeine, creatine, beta-alanine, amino acids, and nitric oxide specialists, the blend of which may inspire a synergistic impact on intense exercise execution and consequent preparing adjustments contrasted with single fixings alone. Thusly, the reason for this article was to survey the hypothetical justification and accessible logical proof evaluating the potential ergogenic estimation of intense and ceaseless ingestion of MIPS, to address potential security concerns encompassing MIPS supplementation, and to feature potential zones for future research. In spite of the fact that immediate correlations between plans of MIPS or between a MIPS and a solitary fixing are testing and regularly unthinkable because of the far reaching utilization of "exclusive mixes" that don't reveal explicit measures of fixings in a given detailing, a significant assemblage of proof proposes that the intense pre-practice utilization of MIPS may decidedly impact solid continuance and abstract temperament, however blended outcomes have been accounted for in regards to the intense impact of MIPS on power and power generation. The interminable utilization of MIPS related to a periodized opposition preparing program seems to enlarge helpful changes in body piece through expanded slender mass gradual addition. Caffeinated beverages and vitality shots are utilitarian enhancements with higher caffeine content advertised toward teenagers and youthful grown-ups with the expectation of boosting vitality. Parallel to its rising fame, there are security worries with the utilization of these drinks. In this, we checked on the effect these enhancements produce on wellbeing and prosperity. Ongoing Findings Most of the advanced advantages of the refreshments can be ascribed to caffeine. The physiologic impacts, assuming any, of different fixings, for example, nutrients and natural concentrates are vague. The nearness of a higher caffeine content when contrasted with sodas may incline to caffeine inebriation in vulnerable people. Caffeinated drinks are every now and again promoted to people inspired by athleti cs and an acti ve way of life. From 2001 to 2008, esti mates of caffeinated savor utilize pre-adult to moderately aged populati ons extended from $24 \%$ to $56 \%$. Most caffeinated drinks highlight caff eine and a blend of different segments, including taurine, sucrose, guarana, ginseng, niacin, pyridoxine, and cyanocobalamin.

\section{Subtopics}

Elements of Multi Ingredient Pre Workout Supplements As the ergogenic impacts of a considerable lot of the essential MIPS fixings have been inspected on an individual premise and assessed somewhere else, the accompanying area will quickly diagram the component of activity, regular dosing conventions, and ergogenic capability of basic MIPS fixings. For the reasons for this survey, the term MIPS will be limited to multi-fixing pre-exercise supplements and isn't to be mistaken for caffeinated drinks, vitality shots, or business sport drinks, which, in spite of making them cover fixings, for example, caffeine, will in general vary in explicit fixing profiles and indicated benefits. ${ }^{1}$

\section{Caffeine}

Caffeine has all the earmarks of being the essential fixing in charge of a few of the intense ergogenic impacts of MIPS, as it is quickly consumed and ordinarily tops in the circulatory system inside $60 \mathrm{~min}$ of ingestion. Caffeine goes about as an adenosine receptor foe and has been appeared to intensely improve discernment just as execution amid continuance, power, and obstruction practice when expended in measurements somewhere in the range of 3 and $6 \mathrm{mg} / \mathrm{kg}$ bodyweight. MIPS definitions that contain somewhere around $300 \mathrm{mg}$ caffeine for each serving will fall inside the worthy dosing range for generally people. ${ }^{2}$ 


\section{Amino acids and amino-containing mixes}

Taurine is an amino-containing sulfonic corrosive that has been accounted for to have cancer prevention agent, metabolic, and ergogenic impacts. While interminable utilization of the substance may improve time-to-weariness amid perseverance work out, intense ingestion of $1.5 \mathrm{~g}$ taurine as a feature of a MIPS has been appeared to improve strong continuance amid opposition work out. Expanded chain amino acids (BCAAs) are regularly added to MIPS details with the goal of boosting rates of muscle protein union, limiting protein breakdown, and lessening exerciseactuated muscle harm. While BCAAs have been estimated to improve weakness, they don't appear to altogether upgrade practice execution or invigorate muscle protein combination. $^{3}$

\section{Nitric oxide operators}

Nitric oxide (NO) is an imperative flagging atom that has been appeared to build blood stream to dynamic muscles, which in principle may result in expanded exercise execution. A few regular segments of MIPS (for example arginine, citrulline) are indicated to expand dimensions of nitric oxide and accordingly improve execution by means of expanded blood stream amid exercise, with obscure execution results revealed in the writing. ${ }^{4}$ Dietary nitrate seems to upgrade intense continuance and high-force practice execution when devoured in portions of $300 \mathrm{mg}$ or higher . Inorganic nitrate (NO3-) is a compound found in beetroot juice and sodium nitrate that is decreased to Nitrite $(\mathrm{NO} 2-)$ in the oral depression by means of enzymatic movement and after that to nitric oxide (NO) in the stomach or fringe tissues under hypoxic conditions. L-arginine is an amino corrosive that is an antecedent required for the blend of nitric oxide. While a few examinations have shown ergogenic benefits coming about because of oral utilization of L-arginine, most of proof proposes that arginine has restricted viability in improving blood stream or exercise execution. L-Citrulline is a superfluous amino corrosive found basically in watermelon that is changed over to Larginine and along these lines advances NO combination. LCitrulline supplementation (regularly joined with malate, a middle of the road in the citrus extract cycle) has been appeared to build vasodilation and improve practice execution when expended constantly in dosages of 6-8 $\mathrm{g}$ for each day. In any case, the dosages found in MIPS are commonly far lower than those that have demonstrated ergogenic impacts. ${ }^{5}$

\section{Creatine}

Creatine is a normally appears in amino corrosive found in the muscle of different creatures. Creatine supplementation is sheltered and has been reliably exhibited to increment intramuscular phosphocreatine levels by $30 \%$ subsequent to enhancing with around $5 \mathrm{~g}(0.03 \mathrm{~g} / \mathrm{kg} /$ portion $)$ every day following a stacking time of $20 \mathrm{~g} / \mathrm{day}(0.3 \mathrm{~g} / \mathrm{kg} / \mathrm{day})$. This routine has gone on to decidedly affect high-force practice execution when constantly devoured in portions equivalent to around 3-5 g for each day following a stacking time of 20 g/day. While post-exercise creatine utilization has all the earmarks of being better than pre-practice supplementation, the customary utilization of something like $3 \mathrm{~g}$ of creatine from a MIPS for each day for 28 days is likely adequate to upgrade practice execution and enlarge preparing adjustments. $^{6}$

\section{Betaine}

Betaine (trimethylglycine) is a normally happening subsidiary of the amino corrosive glycine which may improve practice execution by expanding rates of creatine combination, raising dimensions of blood nitric oxide, and advancing liquid and warm homeostasis. Ceaseless supplementation with $1.25-2.5 \mathrm{~g}$ every day of betaine has been shown to upgrade reiterations to exhaustion and all out volume load finished amid obstruction practice, with enhancements in power and power creation additionally announced. In any case, the measurements utilized in these examinations are far higher than those found in a run of the mill MIPS. ${ }^{7}$

\section{Beta-alanine}

$\beta$-alanine is a typical MIPS segment and forerunner to carnosine, a dipeptide which goes about as an intramuscular support. The utilization of 4-6 g $\beta$-alanine every day over a time of somewhere around about fourteen days has been exhibited to improve high-force practice execution. Consequently, gave that a MIPS contains adequate measures of $\beta$-alanine, comparative ergogenic impacts can be normal, whenever devoured every day to suitably keep up intramuscular carnosine levels. ${ }^{8}$

\section{Reactions of MIPS}

1. Pre-exercises can build overstimulation and jumpiness. The normal pre-exercise recipe concentrates caffeine, pressing in 435 milligrams by and large. The issue is a lot of caffeine, joined with different stimulants and exercise builds the danger of a hazardously high pulse, overexertion, and even damage.

2. Pre-exercises in some cases don't give any profit whatsoever. A huge peril is paying cash for various stimulants that raise your pulse however lead to hazardously hypertension and cardiovascular framework strain .

The issue is ordinarily found in the 'restrictive mix' on the enhancement name. Keeping the pre-exercise definition mystery enables organizations to sneak fixings, for example, pre-hormones, amphetamines, and different synthetic concoctions, past FDA endorsement. For example, the FDA as of late documented criminal allegations against USP Labs, the creators of the pre-exercise supplements Jack3d and OxyElite Pro, whose items have been connected to intense liver poisonous quality and numerous passings.

1. The pre-exercise beta-alanine can cause impermanent skin disturbance. At an appropriate measurement, betaalanine will cause impermanent shivering and irritation of the skin. This is not something to be stressed over and will die down inside 60 minutes. 
2. The pre-exercise L-arginine can cause gastrointestinal problems with high dosages. ${ }^{9}$

\section{Security ramifications of MIPS Use}

To date, significant writing proposes that the utilization of numerous MIPS seems, by all accounts, to be generally sheltered with negligible revealed unfavorable impacts. In any case, most investigations looking at the impacts of MIPS ingestion are moderately short (under about two months). A few investigations have analyzed the impacts of incessant MIPS ingestion on pulse, circulatory strain and a few hematologic markers with negligible unfriendly impacts announced. These impacts of fluctuating terms of MIPS use on wellbeing related results are illustrated underneath in their individual segments. It is fundamental to take note of that numerous examinations just report mean changes inside the whole example and don't determine whether a couple of people surpassed ordinary reaches anytime amid the testing time frame. This announcing method may veil unfriendly occasions if singular changes better than average extents are not unmistakably detailed in a way like that utilized by Jung and associates. ${ }^{10}$ Future examinations should utilize this way to deal with present an increasingly far reaching image of the impacts of MIPS on different clinical markers of security. All things considered, similarly as with numerous physician recommended prescriptions, momentary utilize frequently will in general be moderately protected with couple of genuine unfriendly impacts. Notwithstanding, clinical signs may take months or even a long time to end up present. Thusly, longer-term information is expected to decide potential antagonistic physiologic adjustments because of incessant introduction to MIPS. Past surveys by Eudy et al. ${ }^{11}$ and Maughan et al. ${ }^{68}$ have thoroughly laid out potential unfriendly impacts of oftentimes utilized fixings in different games supplements including MIPS. Concerns in regards to wellbeing of enhancement use go a long ways past only looking at the fixing list that pronounced on the enhancement mark, as a few enhancements have been found to contain contaminants, for example, substantial metals, intense stimulants, or different restricted fixings. Alarmingly, Geyer et al. ${ }^{12}$ revealed that up to $15 \%$ of dietary enhancements contained hormones or prohormones. One especially concerning report distributed in $2014 .{ }^{13}$ showed that a methamphetamine simple was recognized in a mainstream MIPS item, which incited its expulsion from the market according to popular demand of the FDA. 1,3dimethylamylamine (DMAA), a comparable strong sympathomimetic fixing once found in an assortment of preexercise supplements, was similarly expelled from the market by the FDA in the wake of being involved as the reason for six passings and more than 100 revealed sicknesses because of its hindering impact on pulse and cardiovascular capacity. Obviously, a few unregulated DMAA analogs, for example, Octodrine (2-amino-6methylheptane) and 1,4-dimethylamylamine have as of late been recognized in an example of dietary enhancements . Because of the receptive as opposed to proactive nature of enhancement guidelines in the United States, all things considered, consistently developing cycles of exploratory stimulants will be available in an assortment of items as prior renditions of these substances are directed and likely restricted. Consequently, any competitor devouring an enhancement risks coming up short a medication test if segments of the enhancement are restricted by their separate association, which could prompt exclusion from investment or loss of work. Unfriendly impacts from expending an enhancement can result from ingestion of known fixings and furthermore from any obscure contaminants or accidental ingestion of uber portions of the recorded fixings. Many enhancement names list fixings as "exclusive mixes" leaving the purchaser to just think about the amount of a given compound is in the item. This could prompt incidental overdose of different fixings, especially on the off chance that they are likewise being devoured in different items, nourishment things, or dietary enhancements. As referenced before, caffeine is a principle fixing in numerous MIPS and utilization of the substance in high portions, regardless of whether purposeful or unexpected, can result in sickness, heart palpitations, arrhythmias, and cerebral pain. Legitimate measurements of every fixing is basic in accordance with potential execution benefits and absolutely in accordance with unfavorable impacts. At the point when singular fixings found in many enhancements are utilized at suggested measurements, most are all around endured. An all around regarded program, Operation Supplement Safety (OPSS.org) has incorporated a rundown of high hazard supplements that could contain restricted substances, for example, destructive stimulants, anabolic steroids or different hormones that could adversely influence one's wellbeing. The concerned competitor ought to check all enhancement buys utilizing such a technique preceding utilization to guarantee that danger of defilement is limited. ${ }^{14}$

\section{Caffeinated Drink Ingredients Caffeine}

Caffeine is normally found in numerous plants including espresso beans, cacao beans, kola nuts, guarana berries, leaves of tea, and yerba mate . Caffeine is a xanthine alkaloid (1,3,7 trimethylxanthine). Caffeine, the fundamental dynamic element of caffeinated drinks, could be from engineered creation or from common sources, for example, guarana and yerba mate. The all out caffeine content from guarana and yerba mate are not constantly incorporated into the name. After ingestion, caffeine is consumed from the gastrointestinal tract inside 30- $60 \mathrm{~min}$ and achieves top serum focus in $15-45 \mathrm{~min}$. Caffeine has basic comparability to adenosine and the fundamental component of activity of caffeine in advancing mental sharpness and decreasing weariness is accepted to be through a reversible estranging impact on adenosine receptors in the focal sensory system (CNS). Caffeine additionally enacts thoughtful neurons to arrival of catecholamines. At high fixations, it causes preparation of intracellular calcium and restrains explicit phosphodiesterases. Caffeine causes tachycardia, expanded 
circulatory strain, increments in gastric emissions, diuresis, and bronchodilation. The majority of these impacts are more prominent in caffeine-gullible people than the routine purchasers. ${ }^{15}$ Caffeine is widely used by hepatic cytochrome P450 (CYP1A2) proteins by demethylation into paraxanthine $(84 \%)$, theobromine $(12 \%)$, and theophylline (4\%). Caffeine disposal shifts between people because of polymorphisms of these compounds which could clarify the distinctions in individual resistance. Different factors which impact caffeine digestion incorporate age, sex, body weight, hereditary inclination, utilization of different prescriptions, and heart and liver maladies. Negative impacts from caffeine utilization can happen either intensely from over measurements or because of incessant reliance or withdrawal. Intense caffeine inebriation causes emesis, palpitations, arrhythmias, insanity, seizures, stroke, and may result in death. Serum caffeine levels higher than $80 \mu \mathrm{g} / \mathrm{mL}$ is viewed as deadly and a portion as low as 3-10 $\mathrm{g}$ might be hazardous in vulnerable people. The executives of intense caffeine inebriation incorporates quick organization of charcoal and the board of the inconveniences, for example, arrhythmias, seizures, and stroke. In intense serious inebriation, hemodialysis can be a viable treatment. Ingestion of caffeine at high portions constantly can prompt numerous physical and mental side effects, for example, peevishness, nervousness, a sleeping disorder, and migraines. For sound grown-ups, $400 \mathrm{mg}$ of caffeine which is proportionate to $4-$ some espresso has been suggested as the day by day caffeine limit by the FDA. This point of confinement depends on caffeine showing a portion subordinate pharmacokinetics. At low dosages (70- 100 $\mathrm{mg}$ ), caffeine disposal shows a direct example. Be that as it may, at higher portions $(250-500 \mathrm{mg}$ ), its freedom is fundamentally weakened and its disposal half-life is drawn out. The American Academy of Pediatrics (AAP) firmly demoralizes the caffeine utilization by kids and young people because of potential pessimistic consequences for their developing heart and sensory systems. As caffeine is broadly accessible in soda pops and chocolates, consequently unavoidable, the AAP prescribes an all out day by day utilization not surpassing $100 \mathrm{mg}$ for youths 12 to 18 years old. ${ }^{16}$

\section{Taurine}

Taurine (2-aminoethane sulfonic corrosive) is a sulfurcontaining unnecessary amino corrosive present in most of the advertised caffeinated drinks. This amino corrosive is found in most mammalian tissues, especially in high fixations in the CNS, heart, and skeletal muscle. It is gotten from methionine and cysteine. Taurine takes an interest in neuro tweak, solidness of cell layer, and regulation of intracellular calcium. It additionally conjugates with bile salts. Evaluated every day admission is around $400 \mathrm{mg}$ in the all inclusive community. The utilization of taurine as a vitality supplement depends on studies guaranteeing that taurine may have useful activities on heart work. The ergogenic portion of taurine is around $50 \mathrm{mg} / \mathrm{kg}$ and the portion present in most caffeinated drinks offer is much lower. In a deliberate survey with 34 considers examining the impacts of caffeinated drinks on physical impacts of competitors, the taurine measurements in caffeinated drinks went between 71 and $3105 \mathrm{mg}$. An in vitro examination utilizing human myocardium additionally neglected to demonstrate an inotropic impact of taurine. ${ }^{17}$

\section{Natural Ingredients}

Numerous natural substances are added to vitality supplements for different indicated benefits yet really have no characterized physiological job. Despite what might be expected, they can possibly apply undesirable impacts because of medication herb associations and extreme touchiness. Ginseng (Panax ginseng), an East Asian herb, is frequently added to caffeinated drinks for its apparent impacts of expanding vitality and memory and diminishing weariness. Ginsenosides are the dynamic fixings. In spite of its far reaching use in Asian nations as a panacea, the security of ginseng should be explained. Ginseng ingestion may cause looseness of the bowels, rashes, a sleeping disorder, hypertension, and cerebral pain. Ginkgo biloba is generally accepted to help psychological capacities, for example, increment in memory and focus. Notwithstanding, there are reports of draining diathesis and seizure action after its ingestion. Milk thorn (silibinin/silymarin) is included with the indicated activity of hepatic detoxification yet a few CYP cooperations have been noted. ${ }^{18}$

\section{Bioactive Substances}

Fixings, for example, L-carnitine, phenylalanine, D glucuronolactone, inositol, and creatine may have bioactive properties by investment in mediator digestion. They are added to vitality supplements with the proposed advantage of improved muscle digestion. They have hazy utility and are not without undesirable impacts. For instance, at high portions, L-carnitine causes gastrointestinal manifestations, for example, sickness, regurgitating, and the runs and furthermore can encourage or potentiate seizures. ${ }^{19}$

\section{Vitamins}

B complex nutrients including nutrient B12, B6, B3, pantothenic corrosive, and folic corrosive are frequently added to vitality refreshments in amounts far surpassing the suggested recompenses. Some have nutrient $\mathrm{C}$ and $\mathrm{E}$ too. Any profit by expending these nutrients in caffeinated drinks is impossible as most people get adequate amounts in their standard eating regimens. It is frequently asserted without verification that these nutrients enlarge readiness and improve mind-set [20]. The amounts of these nutrients frequently surpass the suggested permitted day by day esteems (DV).

\section{Sugars}

Caffeinated drinks are purposefully made sweet to pull in the more youthful populace. The typical wellsprings of sugar incorporate glucose, high-fructose corn syrup, sucrose, and maltodextrin. A couple are improved with fake sugars for low-calorie alternatives. 


\section{Different Ingredients}

Most contain sodium, potassium, calcium, and magnesium in little sums. Different additives, shading specialists, and emulsifiers are additionally included.

\section{Liquor and Energy Drinks}

The utilization of caffeinated drinks in early youthfulness may go about as a harbinger for expanded danger of liquor use further down the road. Teenagers expending both liquor and caffeinated drinks experience school issues such astruancy, lack of engagement in school, poor scholastic accomplishments, and harassing when contrasted with companions who devour either liquor or caffeinated drinks alone and furthermore to non-purchasers of either drink. Despite the fact that the premixed energized mixed refreshments were pulled off the market by the FDA, the act of blending liquor with caffeinated drinks is broadly predominant. ${ }^{22}$ A national study found that $13 \%$ of center and secondary school understudies devoured liquor blended with caffeinated drinks at any rate once in the earlier year. For youthful grown-ups, this extent was significantly higher at simply over $33 \%$. One examination noticed that the lifetime utilization of caffeinated drink blended with liquor was $75 \%$ among college understudies. The stimulant impact of caffeine in the caffeinated beverages can alienate the depressant impact of liquor. This decreases the hindrance of equalization and laziness incited by liquor bringing about diminished dimension of apparent inebriation The accomplishment of this "wide alert alcoholic state" may prompt genuine unfriendly results, for example, hitting the bottle hard and driving while inebriated. Numerous people report blending liquor in caffeinated beverages to shroud the flavor of the liquor and apparently prompting progressively quick and inordinate liquor admission. Routine caffeinated drink clients who create sleep deprivation and daytime exhaustion may likewise devour liquor normally to counter these impacts. A higher commonness of unprotected sex and an expansion in rapes (both danger of manhandling and being mishandled) happens in the individuals who devour blended liquor and caffeinated drinks. A few nations commit the producers of caffeinated beverages to show that the beverages ought not be overwhelmed by liquor. Greasy liver infection is another potential worry because of the added substance impacts of liquor and sugar from the caffeinated drinks. ${ }^{23}$

\section{Harmful Effects of Energy Drinks}

As the prominence of caffeinated drinks keeps on rising, it is vital to think about their capability to cause antagonistic impacts. Five separate instances of seizures have been accounted for to be related with the utilization of caffeinated drinks. There have additionally been 4 reports of mental screech after ingestion of caffeinated drinks by patients with known mental sickness. Other unfavorable impacts suspected to be connected to caffeinated drinks incorporate expanded pulse and circulatory strain, heart failure, supraventricular tachycardia, postural tachycardia, and stroke.75- 80 One instance of hypersensitivity after utilization of a caffeinated drink has likewise been reported.81Caffeine has been accounted for to cause a sleeping disorder, anxiety, arrhythmias, osteoporosis, cardiovascular sickness, frailty, pregnancy and labor confusions, gastrointestinal surprise, and passing. Different concerns have been communicated in regards to inconvenient impacts of caffeine when utilized with liquor, nicotine, or unlawful substances.1,82Another purpose of concern in regards to caffeine is its capacity to cause withdrawal side effects (ie, migraines, weakness, and dormancy) on suspension. In routine caffeine clients, execution upgrading impacts might be blunted. In moderate amounts, caffeine $(3-6 \mathrm{mg} / \mathrm{kg} / \mathrm{day})$ can be ergogenic and isn't for the most part connected with antagonistic impacts. Despite the fact that an accord has not been set up, Ganio et $\mathrm{al}^{24}$ prescribe competitors to consider avoiding caff eine ingestion for no less than 7 days before an occasion to take into consideration withdrawal side effects to die down and for adenosine receptor down guideline to happen. The US Food and Drug Administration (FDA) controls soda pops as nourishments and limits caffeine to $0.02 \%$ or $71 \mathrm{mg} / 12$ liquid oz.1 Aside from a little bunch of low-caffeine options, most caffeinated drinks surpass as far as possible. Reissig et all as of late covered caffeinated drinks containing as much as $505 \mathrm{mg}$ of caffeine in a solitary holder-the caffeine proportional to drinking 14 jars of Coca-Cola. This is on the grounds that caffeinated drink makers guarantee their items ought to be delegated dietary enhancements under the 1994 Dietary Supplement Health and Education Act. These "useful sustenances" right now dwell in an administrative hazy area, in which producers make structure-work claims, however all the while keep away from the dis-claimer required for enhancements and the caffeine impediments doled out to nourishments. Additionally, most makers have not sought after intentional affirmation programs accessible for dietary supple-ments (eg, National Sanitation Foundation International, United States Pharmacopeia, Natural Products Association). The absence of guideline encompassing these exceptionally charged caffeinated drinks as of late incited a formal solicitation to the FDA by a gathering of 100 doctors, drug specialists, and researchers with showed aptitude in the fi eld.83Few unfavorable impacts are related with taurine in amounts that are found in caffeinated drinks. An investigation in hemodialysis patients accepting taurine 100 $\mathrm{mg} / \mathrm{kg} /$ day was stopped after 4 patients were enrolled on account of watched gathering (2 patients), discombobulation ( 2 patients), and renal transplantation ( 1 patient).84 Taurine has additionally been found to instigate hypothermia and hyperkalemia in patients with uncompensated adrenocortical insuffi ciency. Different reports incorporate tingling in psoriasis patients with $2 \mathrm{~g} /$ day; sickness, migraine, dazedness, and step unsettling influences in epilepsy patients with $1.5 \mathrm{~g} / \mathrm{day}$; and mellow looseness of the bowels and blockage with oral taurine.85 Adverse eff ects detailed for ginseng incorporate sleep deprivation, mastalgia, vaginal dying, tachycardia, craziness, cerebral arteritis, StevensJohnson disorder, cholestatic hepatitis, amenorrhea, 
diminished hunger, edema, fever, pruritus, hypotension, palpitations, cerebral pain, vertigo, elation, and neonatal death.86Niacin is commonly very much endured when ingested in the sums found in caffeinated drinks. Flushing can happen rearward er admission of $30 \mathrm{mg}$ for each day, and hepatotoxicity may happen at portions $3 \mathrm{~g} / \mathrm{day}$. Expansive dosages may likewise cause gastrointestinal unsettling influences, weakened glucose resistance, hyperuricemia, rashes, wooziness, tiredness, and migraines. Pyridoxine can cause gastrointestinal unsettling influences, cerebral pain, paresthesia, lethargy, diminished serum folic corrosive, unfavorably susceptible responses, bosom soreness/amplification, and photosensitivity. Cyanocobalamin does not regularly causes unfavorable impacts, however looseness of the bowels, thrombosis, rash, and hypersensitivity have been reported.86Aft er the market evacuation of ephedra in 2004, unpleasant orange rose as a substitution and might be found in some caffeinated drinks. The dynamic fixings in severe orange enhancements are synephrine and octopamine, which enact $\alpha$-adrenergic receptors. Some unfriendly impacts related with harsh orange incorporate ischemic stroke, work out initiated syncope, QT prolongation, myocardial dead tissue, and expanded circulatory strain. At the point when joined with other sympathomimetic fixings (eg, caffeine, guarana), unpleasant orange may cause added substance stimulant effects. $^{25}$

\section{Correlation}

The best alternative: Multi Ingredient Pre Workout

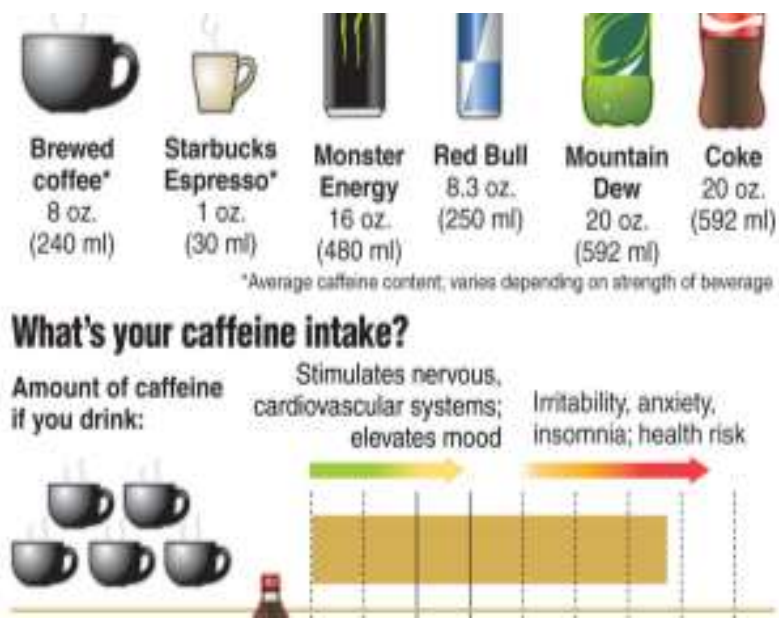

Fig. 1: Multi Ingredient Pre Workout

\section{Pre Workout Benefits}

1. Raise Energy Levels

2. Increase Focus

3. Extend Stamina and Power

Pre Workout supplements have turned out to be prominent lately as a protected method to expand your exercises and get the exceptionally most out of your instructional meetings.
They normally consolidate a stimulant, for example, Caffeine with various different fixings to give your body the fuel it needs to perform getting it done amid an exercise.

The best pre exercises will consolidate a little portion of Caffeine with fixings, for example, Creatine, L-Citrulline and L-Theanine to make you feel taking care of business while at the rec center.

There are a few enhancements which are over-burden with stimulants. It's ideal to maintain a strategic distance from these in view of the symptoms they can cause. A little portion of Caffeine ought to be sufficient to help your exercise.

The most noticeably awful alternative: Energy Drink

\section{Caffeinated Drink Benefits \\ Raise Energy Levels}

That expedites us to our least-favored alternative - the Energy Drink. Obviously, this will be a lot of individuals' most loved pre exercise custom. Yet, as we would like to think, the dangers exceed the advantages.

Obviously, an Energy Drink is going to help raise your vitality levels in light of the fact that the greater part of them use stimulants, for example, Caffeine.

The issue here is that numerous Energy Drinks utilize an abundant excess Caffeine, and that can cause dreadful reactions, for example, vitality accidents of some anxiety.

Besides, Energy Drinks are generally stuffed with sugar or different sugars. Regardless of whether they guarantee not to utilize sugar or be 'without sugar', they're probably going to utilize some frightful synthetic substances or counterfeit sugars to improve the taste. This isn't great, the greatest number of fake sugars can cause reactions.

Our recommendation is to avoid Energy Drinks through and through, and that implies before your exercise as well. ${ }^{26}$

\section{Conclusion}

1. Excess of everything is bad though take it in right amount and don't make it a habit.

2. Fight for fitness without the use of energy drinks.

3. Energy drinks boost energy just for a period of time

4. The power surge of sugar usually last about 30mins to one hour and caffeine about 2 hours and usually followed by low energy.

5. Do not get too much dependent on supplements as they increase the cholestrol level and blood pressure.

\section{Conflict of Interest: None.}

\section{Reference}

1. Campbell B, Wilborn C, La Bounty P, Taylor L, Nelson MT, Greenwood M et al. International Society of Sports Nutrition position stand: energy drinks. J Int Soc Sports Nutr 2013;10(1):1.

2. Goldstein ER, Ziegenfuss T, Kalman D, Kreider R, Campbell $\mathrm{B}$, Wilborn $\mathrm{C}$ et al, International society of sports nutrition position stand: caffeine and performance. J Int Soc Sports Nutr 2010;7(1):5. 
3. Wolfe RR. Branched-chain amino acids and muscle protein synthesis in humans: myth or reality? J Int Soc Sports Nutr 2017;14:30.

4. Bescos R, Sureda A, Tur JA, Pons A. The effect of nitricoxide-related supplements on human performance. Sports Med 2012;42(2):99-117.

5. Figueroa A, Wong A, Jaime SJ, Gonzales JU. Influence of Lcitrulline and watermelon supplementation on vascular function and exercise performance. Curr Opin Clin Nutr Metab Care 2017;20(1):92-8.

6. Kreider RB, Kalman DS, Antonio J, Ziegenfuss TN, Wildman $\mathrm{R}$, Collins R et al, International Society of Sports Nutrition position stand: safety and efficacy of creatine supplementation in exercise, sport, and medicine. J Int Soc Sports Nutr 2017;14(1): 18

7. Lee EC, Maresh CM, Kraemer WJ, Yamamoto LM, Hatfield DL, Bailey BL et al. Ergogenic effects of betaine supplementation on strength and power performance. J Int Soc Sports Nutr 2010;7(1):27.

8. Trexler ET, Smith-Ryan AE, Stout JR, Hoffman JR, Wilborn $\mathrm{CD}$, Sale $\mathrm{C}$ et al, International society of sports nutrition position stand: Beta-alanine. J Int Soc Sports Nutr 2015;12(1):30.

9. https://healthtrends.com/pre-workout/

10. Jung YP, Earnest CP, Koozehchian M, Cho M, Barringer N, Walker D et al, Effects of ingesting a pre-workout dietary supplement with and without synephrine for 8 weeks on training adaptations in resistance-trained males. J Int Soc Sports Nutr 2017;14(1):1.

11. Eudy AE, Gordon LL, Hockaday BC, Lee DA, Lee V, Luu D et al, Efficacy and safety of ingredients found in preworkout supplements. Am J Health Syst Pharm 2013;70(7):577-88.

12. Geyer H, Parr MK, Mareck U, Reinhart U, Schrader Y, Schanzer W et al. Analysis of non-hormonal nutritional supplements for anabolic-androgenic steroids results of an international study. Int J Sports Med 2004;25(2):124-9.

13. Cohen PA, Travis JC, Venhuis BJ. A methamphetamine analog (N,alpha-diethylphenylethylamine) identified in a mainstream dietary supplement. Drug Test Anal 2014;6(78):805-7.

14. Kaplan GB, Greenblatt DJ, Ehrenberg BL, Goddard JE, Cotreau MM, Harmatz JS et al, Dose-dependent pharmacokinetics and psychomotor effects of caffeine in humans. J Clin Pharmacol 1997;37(8):693-703.

15. Heckman MA, Weil J, de Mejia EG. Caffeine (1, 3, 7 trimethylxanthine) in foods: a comprehensive review on consumption, functionality, safety, and regulatory matters. $J$ Food Sci 2010;75(3)

16. https://www. aap.org/en-us/about-the-aap/aap-pressroom/pages/kids-shouldnot-consume-energy-drinks,-andrarely-need-sports-drinks,-saysaap.aspx/

17. Chaban R, Kornberger A, Branski N, Buschmann K, Stumpf $\mathrm{N}$, Beiras-Fernandez A, et al. In-vitro examination of the positive inotropic effect of caffeine and taurine, the two most frequent active ingredients of energy drinks. BMC Cardiovasc Disord 2017;17(1):1-8.

18. WolkBJ, GanetskyM, BabuKM.Toxicityofenergydrinks. Curr Opin Pediatr 2012;24(2):243-51.

19. Seifert SM, Schaechter JL, Hershorin ER, Lipshultz SE. Health effects of energy drinks on children, adolescents, and Young Adults. Pediatr 2011;127(3):511-28.

20. Heckman MA, Sherry K, de Mejia EG. Energy drinks: an assessment of their market size, consumer demographics, ingredient profile, functionality, and regulations in the United States. Compr Rev Food Sci Food Saf 2010;9(3):303-17.

21. Stephanie L. Ballard, , BCPS; Jennifer J. Wellborn-Kim, , BCPS; Kevin A. Clauson, Effects of Commercial Energy Drink Consumption on Athletic Performance and Body Composition, The Physician and Sportsmedicine ISSN 0091-3847, 2010;1(38).

22. Berger L, Fendrich M, Fuhrmann D. Alcohol mixed with energy drinks: are there associated negative consequences beyond hazardous drinking in college students? Addict Behav 2013;38(9): 2428-32.

23. ReisR,CharehsazM,SipahiH,EkiciAID,MacitÇ,AkkayaH,et al. Energy drink induced lipid peroxidation and oxidative damage in rat liver and brain when used alone or combined with alcohol. J Food Sci 2017;82(4):1037-43.

24. Ganio MS, Klau JF, Casa DJ, Armstrong LE, Maresh CM. Eff ect of caff eine on sport-specifi c endurance performance: a systematic review. J Strength Cond Res 2009;23(1):315-324.

25. Clauson KA, Shields KM, McQueen CE, Persad N. Safety issues associ-ated with commercially available energy drinks. $J$ Am Pharm Assoc 2008;48(3):e55-e63; e64-e67.

26. https://www.thesportreview.com/health-and-fitness/preworkout-vs-coffee-energy-drink/

How to cite this article: Bhasin T. Multi-Ingredient PreWorkout Supplements and Energy Drinks. J Nutr, Metab Health Sci, 2019;2(2):23-9. 